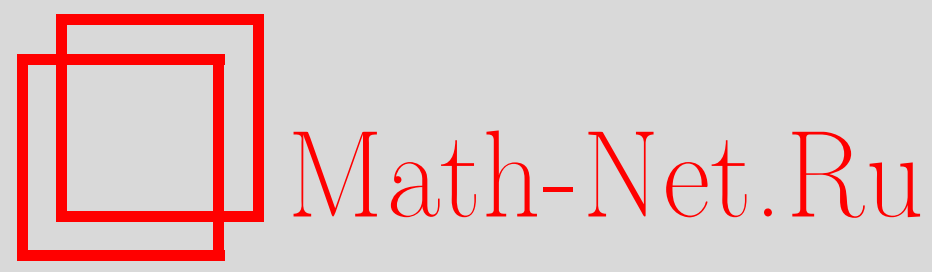

А. А. Туганбаев, Кольца, над которыми все конечно порожденные модули $\aleph_{0}$-инъективны, Дискрет. матем., 2009, том 21, выпуск 4, 76-81

DOI: https://doi.org/10.4213/dm1072

Использование Общероссийского математического портала Math-Net.Ru подразумевает, что вы прочитали и согласны с пользовательским соглашением http://www . mathnet.ru/rus/agreement

Параметры загрузки:

IP : 52.87 .193 .239

26 апреля 2023 г., $15: 27: 10$ 
УДК 512.62

\section{Кольца, над которыми все конечно порожденные модули $\boldsymbol{\aleph}_{0}$-инъективны}

() 2009 г. А. А. Туганбаев

Доказано, что все конечно порожденные правые $A$-модули $\aleph_{0}$-инъективны в точности тогда, когда $A-\aleph_{0}$-инъективное справа регулярное кольцо.

Все кольца предполагаются ассоциативными и с ненулевой единицей, модули предполагаются унитарными. Хорошо известен следующий результат.

Теорема А ([4]). Для кольца А равносильны условия:

(1) все конечно порожденные правые А-модули инъективны;

(2) A- полупростое кольц̧о.

Пусть $M$ - правый модуль над кольцом $A$ и $\boldsymbol{\aleph}$ - кардинальное число. Модуль $M$ называется $\aleph$-инъективным, если для любого $\aleph$-порожденного правого идеала $B$ кольца $A$ каждый гомоморфизм $B_{A} \rightarrow M$ продолжается до гомоморфизма $A_{A} \rightarrow M$. Кольцо $A$ называется регулярным (по фон Нейману), если $a \in a A a$ для любого элемента $a \in A$.

Основным результатом данной работы является теорема 1.

Теорема 1. Для кольца А равносильны условия:

(1) все конечно порожденные правые А-модули $\boldsymbol{\aleph}_{0}$-инъективны;

(2) $A$ - регулярное кольцуо и в А для любого счетного множества $\left\{e_{i}\right\}_{i=1}^{\infty}$ ортогональных идемпотентов и каждого счетного множества $\left\{a_{i}\right\}_{i=1}^{\infty}$ элементов найдется такой элемент $a \in A$, что ае $i=a_{i} e_{i}$ для всех $i$.

Замечание 1. В связи с теоремой А и теоремой 1 заметим, что существуют неартиновы кольца $A$, удовлетворяющие теореме 1 . Например, можно взять

$$
A=\prod_{i=1}^{\infty} A_{i},
$$

где все $A_{i}$ - поля (см. лемму 3 данной работы).

Доказательство теоремы 1 разбито на ряд утверждений, некоторые из которых представляют самостоятельный интерес. Приведем необходимые определения и обозначения.

Модуль $M$ называется полупростым, если все его подмодули являются прямыми слагаемыми в $M$. Ненулевые полупростые модули совпадают с прямыми суммами простых 
модулей. Полупростые справа (слева) кольца совпадают с кольцами вида $A_{1} \times \ldots \times A_{n}$, где каждое кольцо $A_{i}$ изоморфно кольцу матриц над некоторым телом. Модуль $M$ называется регулярным, если все его конечно порожденные подмодули являются прямыми слагаемыми в $M$. Кольцо $A$ является регулярным в точности тогда, когда модуль $A_{A}\left({ }_{A} A\right)$ регулярен. Регулярные кольца, в которых все идемпотенты центральны, называются строго регулярными. Кольцо $A$ строго регулярно в точности тогда, когда $a \in a^{2} A\left(a \in A a^{2}\right)$ для любого элемента $a \in A$. Модуль $M$ называется проективным, если для любого модульного эпиморфизма $h: X \rightarrow \bar{X}$ и каждого гомоморфизма $\bar{f}: M \rightarrow \bar{X}$ существует такой гомоморфизм $f: M \rightarrow X$, что $\bar{f}=h f$. Модуль $M$ называется инъективным, если для любого модульного мономорфизма $h: \bar{X} \rightarrow X$ и каждого гомоморфизма $\bar{f}: \bar{X} \rightarrow M$ существует такой гомоморфизм $f: X \rightarrow M$, что $\bar{f}=f h$.

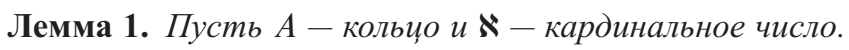

(1) Если $\left\{M_{i}\right\}_{i \in I}$ - некоторое множество ふ-инъективных правых $A$-модулей, то $\prod_{i \in I} M_{i}-\mathbf{\aleph}$-инъективный модуль.

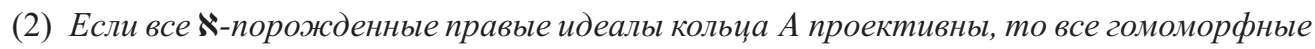

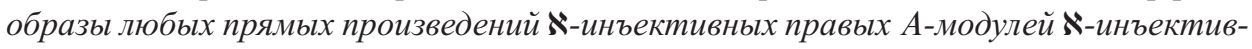
$H b l$.

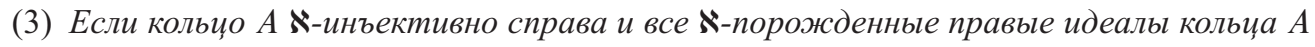
проективны, то все конечно порожденные правые А-модули ґ-инъективны.

Доказательство. 1. Пусть $\pi_{i}: \prod_{i \in I} M_{i} \rightarrow M_{i}-$ естественные проекции, $\mathbf{\aleph}-$ кардинальное число, $B$ - א-порожденный правый идеал кольца $A$ и $f: B \rightarrow M$ - гомоморфизм правых $A$-модулей. Так как все модули $M_{i}$ ※-инъективны, то гомоморфизмы $\pi_{i} f: B \rightarrow M_{i}$ продолжаются до гомоморфизмов $g_{i}: A_{A} \rightarrow M_{i}$. Тогда гомоморфизм $\prod_{i \in I} g_{i}: A_{A} \rightarrow \prod_{i \in I} M_{i}$ - продолжение гомоморфизма $f$. Поэтому $\prod_{i \in I} M_{i}-$ ぶ-инъективный модуль.

2. Пусть $\left\{M_{i}\right\}_{i \in I}$ - некоторое множество $\aleph$-инъективных правых $A$-модулей, $M=\prod_{i \in I} M_{i}, h: M \rightarrow \bar{M}-$ эпиморфизм, $B-\aleph$-порожденный правый идеал в $A$ и $\bar{f}: B \rightarrow \bar{M}$ - гомоморфизм правых $A$-модулей. Так как $B-$ проективный $A$-модуль, то $\bar{f}=h f$ для некоторого гомоморфизма $f: B_{A} \rightarrow M$. По п. $1 M-\aleph$-инъективный правый $A$-модуль. Поэтому гомоморфизм $f$ продолжается до некоторого гомоморфизма $g: A_{A} \rightarrow M$. Тогда $h g: A_{A} \rightarrow \bar{M}$ - продолжение гомоморфизма $\bar{f}$. Поэтому модуль $\bar{M}$ ґ-инъективен.

Пусть $M-$ конечно порожденный правый $A$-модуль. Существует такое конечное множество $\left\{A_{i}\right\}_{i=1}^{n}$ изоморфных копий свободного циклического модуля $A_{A}$, что $M-$ гомоморфный образ модуля $\bigoplus_{i=1}^{n} A_{i}$. Так как

$$
\bigoplus_{i=1}^{n} A_{i}=\prod_{i=1}^{n} A_{i},
$$

то по п. $2 M-\aleph$-инъективный модуль.

Лемма 2 ([3, 2.14, 2.15]). Пусть $A$ - регулярное кольцุо и $B-\boldsymbol{\aleph}_{0}$-порожденный правый идеал в А. Тогда правый $A$-модуль $B$ проективен и существует такое счетное множество $\left\{e_{i}\right\}_{i=1}^{\infty}$ ортогональных идемпотентов кольиа $A$, что

$$
B=\bigoplus_{i=1}^{\infty} e_{i} A .
$$


Лемма 3. Для кольија А равносильны условия:

(1) все конечно порожденные правые А-модули $\aleph_{0}$-инъективны;

(2) все циклические правые А-модули $\boldsymbol{\aleph}_{0}$-инъективны;

(3) $A-\aleph_{0}$-инъективное справа регулярное кольияо;

(4) кольцо А изоморфно прямому произведению $\aleph_{0}$-инъективных справа регулярных колец.

Доказательство. Импликации (1) $\Rightarrow(2)$ и (3) $\Rightarrow$ (4) очевидны.

$(2) \Rightarrow(3)$. Так как модуль $A_{A}$ цикличен, то по (2) кольцо $A \aleph_{0}$-инъективно справа. Пусть $a \in A$ и $f: a A \rightarrow a A \subseteq A-$ тождественное отображение. Так как циклический модуль $а A \boldsymbol{\aleph}_{0}$-инъективен, то $f$ продолжается до гомоморфизма $g: A_{A} \rightarrow a A$. Тогда $a A=g(A), g^{2}=g, a A-$ прямое слагаемое модуля $A_{A}$, и кольцо $A$ регулярно.

$(4) \Rightarrow(3)$. Так как кольцо $A$ изоморфно прямому произведению регулярных колец, то $A$ регулярно. С помощью леммы 1 (1) проверяется, что $A-\aleph_{0}$-инъективное справа кольцо.

(3) $\Rightarrow$ (1). По лемме 2 все $\aleph_{0}$-порожденные правые идеалы кольца $A$ проективны. По лемме 1 (3) все конечно порожденные правые $A$-модули $\aleph_{0}$-инъективны.

Лемма 4. Для регулярного кольца А равносильньл условия:

(1) $A-\boldsymbol{\aleph}_{0}$-инъективное справа кольияо;

(2) в А для любого счетного множества $\left\{e_{i}\right\}_{i=1}^{\infty}$ ортогональных идемпотентов $u$ каждого счетного подмножества $\left\{a_{i}\right\}_{i=1}^{\infty}$ найдется такой элемент $a \in A$, что $a e_{i}=a_{i} e_{i}$ для всех $i$.

Доказательство. (1) $\Rightarrow$ (2). Существует такой гомоморфизм $f: \bigoplus_{i=1}^{\infty} e_{i} A \rightarrow A_{A}$, что $f\left(\sum e_{i} x_{i}\right)=\sum a_{i} e_{i} x_{i}$. По условию, $f$ продолжается до гомоморфизма $g: A_{A} \rightarrow A_{A}$. Обозначим $a=g(1)$. Тогда $a e_{i}=a_{i} e_{i}$ для всех $i$.

$(2) \Rightarrow(1)$. Пусть $B-\aleph_{0}$-порожденный правый идеал кольца $A$ и $f: B \rightarrow A-$ гомоморфизм правых $A$-модулей. По лемме 2 существует такое счетное множество $\left\{e_{i}\right\}_{i=1}^{\infty}$ ортогональных идемпотентов кольца $A$, что

$$
B=\bigoplus_{i=1}^{\infty} e_{i} A
$$

Обозначим $a_{i}=f\left(e_{i}\right)$. Тогда

$$
f\left(e_{i}\right)=f\left(e_{i}\right) e_{i}=a_{i} e_{i}, \quad i=1,2, \ldots
$$

По (2) найдется такой элемент $a \in A$, что $a e_{i}=a_{i} e_{i}=f\left(e_{i}\right)$ для всех $i$. Определим гомоморфизм $g: A_{A} \rightarrow A_{A}$ равенством $g(x)=a x, x \in A$. Пусть

$$
b=\sum_{i=1}^{n} e_{i} x_{i} \in B, \quad x_{i} \in A .
$$


Тогда

$$
g(b)=a b=\sum_{i=1}^{n} a e_{i} x_{i}=\sum_{i=1}^{n} f\left(e_{i}\right) x_{i}=f(b) .
$$

Поэтому $g$ - продолжение $f$ и кольцо $A \aleph_{0}$-инъективно справа.

Теорема 1 вытекает из леммы 3 и леммы 4.

Если в теореме 1 заменить конечно порожденные модули на $\boldsymbol{\aleph}_{0}$-порожденные, то придем к полупростым кольцам.

Замечание 2. Для кольца $A$ равносильны условия:

(1) все $\aleph_{0}$-порожденные правые $A$-модули $\aleph_{0}$-инъективны;

(2) в кольце $A$ все $\aleph_{0}$-порожденные правые идеалы $\aleph_{0}$-инъективны;

(3) $A$ - регулярное кольцо без бесконечных множеств ортогональных идемпотентов;

(4) $A$ - полупростое кольцо;

(5) все правые $A$-модули и все левые $A$-модули инъективны и проективны.

Доказательство. Эквивалентность условий (3), (4) и (5 хорошо известна (см. [2, 19.26А] и $[1,8.2 .2])$. Импликации (4) $\Rightarrow(1)$ и (1) $\Rightarrow(2)$ очевидны.

$(2) \Rightarrow(3)$. По теореме 1 кольцо $A$ регулярно. Допустим, что $A$ содержит некоторое бесконечное множество $\left\{e_{i}\right\}_{i=1}^{\infty}$ ненулевых ортогональных идемпотентов. Обозначим через $E$ правый идеал $\bigoplus_{i=1}^{\infty} e_{i} A$ кольца $A$. Пусть $f: B \rightarrow B \subseteq A$ - тождественное отображение. Так как $\aleph_{0}$-порожденный правый идеал $B \boldsymbol{\aleph}_{0}$-инъективен, то $f$ продолжается до гомоморфизма $g: A_{A} \rightarrow B$. Тогда $B=g(A), g^{2}=g, B-$ прямое слагаемое модуля $A_{A}$ и кольцо $A$ полупросто.

Наша следующая цель - показать, что $\aleph_{0}$-инъективные справа регулярные кольца совпадают с $\aleph_{0}$-алгебраически компактными справа регулярными кольцами (см. предложение 1 ниже).

Пусть $M$ - правый модуль над кольцом $A$ и $L\left(a_{i j}, m_{i}, M_{A}\right)$ - система из счетного числа линейных уравнений $\left\{\sum_{j=0}^{t(i)} x_{j} a_{i j}=m_{i}\right\}_{i=0}^{\infty}$ с коэффициентами $a_{i j} \in A$ от счетного числа неизвестных $\left\{x_{j}\right\}_{j=0}^{\infty}$, принимающих значения в $M$, причем любая конечная подсистема этой системы разрешима. Такая система $L\left(a_{i j}, m_{i}, M_{A}\right)$ называется счетной конечно разрешимой системой. Правый $A$-модуль $M$ называется $\boldsymbol{\aleph}_{0}$-алгебраически компактным, если любая счетная конечно разрешимая система $L\left(a_{i j}, m_{i}, M_{A}\right)$ имеет решение в $M$.

Лемма 5. Для правого модуля $М$ над кольцчом А равносильны условия:

(1) $М-\aleph_{0}$-алгебраччески компактный модуль;

(2) для любой счетной конечно разрешимой системы $L\left(a_{i j}, m_{i}, M_{A}\right)=L$ найдется такой элемент $y_{0} \in M$, что каждая конечная подсистема $L^{*}$ в L имеет частное решение $\bar{y}^{*}=\left\{y_{0}, y_{1}^{*}, \ldots\right\}$, где $y_{0}$ зависит только от системы L. 
Доказательство. Импликация (1) $\Rightarrow(2)$ проверяется непосредственно.

$(2) \Rightarrow(1)$. Необходимо доказать существование решения $\bar{y}=\left\{y_{0}, y_{1}, \ldots\right\}$ системы $L$. Существование элементов $y_{n} \in M$ докажем по индукции. По условию $y_{0}$ существует. Допустим, что $y_{0}, \ldots, y_{n}$ найдены. Рассмотрим систему

$$
N \equiv\left\{\sum_{j=n+1}^{t(i)} x_{j} a_{i j}=m_{i}-\sum_{j=0}^{n} y_{j} a_{i j}\right\}_{i=0}^{\infty} .
$$

По условию, примененному к $N$ и неизвестному $x_{n+1}$, найдется $y_{n+1} \in M$, для которого любая конечная подсистема $N^{*}$ в $N$ имеет решение $\bar{z}^{*} \equiv\left\{y_{n+1}, y_{n+2}^{*}, \ldots\right\}$, где $y_{n+1}$ зависит от системы $N$, но $y_{n+1}$ не меняется при переходе к другой конечной подсистеме из $N$. Докажем, что $\bar{y} \equiv\left\{y_{0}, y_{1}, \ldots\right\}$ - требуемое решение системы $L$. Достаточно доказать, что $\bar{y}$ - решение любой конечной подсистемы $L^{*}$ из $L$. Подсистема $L^{*}$ содержит лишь конечное число неизвестных $x_{0}, \ldots, x_{n}$. Из способа выбора последовательности $\left\{y_{m}\right\}_{m=0}^{\infty}$ следует существование такого решения $\bar{y}^{*}$ системы $L^{*}$, что $y_{0}, \ldots, y_{n} \in M-$ первые $n+1$ компонент этого решения. Поэтому $\bar{y}-$ решение системы $L^{*}$.

Предложение 1. Для регулярного кольияа А равносильны условия:

(1) $A-\aleph_{0}$-инъективное справа кольио;

(2) $A-\aleph_{0}$-алгебраически компактное справа кольцуо.

Доказательство. (1) $\Rightarrow$ (2). Пусть $L$ - такая счетная конечно разрешимая система $\left\{\sum_{j=0}^{t(i)} x_{j} a_{i j}=m_{i}\right\}_{i=0}^{\infty}$, что $a_{i j} \in A, m_{i} \in A$, и для любого $n \geqslant 0$ конечная подсистема

$$
L_{n} \equiv\left\{\sum_{j=0}^{t(i)} x_{j} a_{i j}=m_{i}\right\}_{i=0}^{n}
$$

в $L$ имеет частное решение $\overline{x(n)}=\left\{x_{j}(n)\right\}_{j=0}^{m(n)}$, где

$$
m(n)=\max _{0 \leqslant i \leqslant n}\{t(i)\} .
$$

По лемме 4 достаточно доказать существование такого множества $\left\{\overline{y(n)}_{n=0}^{\infty}\right\}$ частных решений системы $L_{n}$, что $\overline{y(n)}=\left\{b, y_{1}(n), y_{2}(n), \ldots\right\}$, где $b$ зависит только от $L$ и не меняется с изменением $n$. Зафиксируем число $n$. Пусть

$$
H_{n} \equiv\left\{\sum_{j=0}^{t(i)} x_{j} a_{i j}=0\right\}_{i=0}^{n}
$$

- однородная конечная система, соответствующая неоднородной системе $L_{n}, G_{n}-$ множество всех решений системы $H_{n}$, и $T_{n}$ - левый идеал в $A$, порожденный всеми первыми компонентами из $G_{n}$. Тогда $G_{n}$ - ядро эндоморфизма $h$ свободного левого $A$-модуля $F$ ранга $m(n)$. Модуль $F$ регулярен (см. [3, 2.11]). Поэтому $h(F)-$ прямое слагаемое в $F$ и модуль $G_{n}$ конечно порожден, поскольку модуль $G_{n}$ изоморфен прямому слагаемому конечно порожденного модуля $F$. Левый идеал $T_{n}$ регулярного кольца $A$ - гомоморфный образ модуля $G_{n}$ и поэтому конечно порожден. Тогда $A=T_{n} \oplus A f_{n}$, где $f_{n}-$ идемпотент. Будем менять $n$. Так как $T_{n} \supseteq T_{n+1}$, то найдется такой идемпотент $e_{n+1}$, что 
$T_{n}=T_{n+1} \oplus A e_{n+1}$ и $f_{n}=f_{n+1}+e_{n+1}$. Обозначим $e_{0}=f_{0}$. Тогда $\left\{e_{i}\right\}_{i=0}^{\infty}-$ счетное множество ортогональных идемпотентов и $f_{n}=\sum_{i=0}^{n} e_{i}$ при $n \geqslant 0$. Обозначим $a_{i}=x_{0}(i) \in A$. Тогда $a_{i}-a_{n} \in T_{i}$ при $i \leqslant n$, поскольку $\overline{x(i)}-\overline{x(n)} \in H_{i}$. Поэтому $a_{i} f_{i}=a_{n} f_{i}$ при $i \leqslant n$. По теореме 1 найдется элемент $b \in A$, для которого $b e_{i}=a_{i} e_{i}$ для всех $i$. Тогда

$$
\begin{gathered}
\left(b-a_{n}\right) f_{n}=\sum_{i=0}^{n}\left(b-a_{n}\right) e_{i}=\sum_{i=0}^{n}\left(b-a_{i}\right) e_{i}=0, \\
\left(b-a_{n}\right)\left(1-f_{n}\right)=b-a_{n}, \quad b-a_{n} \in T_{n} .
\end{gathered}
$$

Поэтому для всех $i$ найдется такое решение $\overline{z(n)}=\left\{z_{j}(n)\right\}_{j=0}^{m(n)}$ однородной системы $H_{n}$, что $b=a_{n}+z_{0}(n)$. Если $\overline{y(n)}$ - сумма решения $\overline{x(n)}$ неоднородной системы $L_{n}$ и решения $\overline{z(n)}$ однородной системы $H_{n}$, то $\overline{y(n)}-$ решение неоднородной системы $L_{n}$ и $\overline{y(n)}=\left\{b, y_{1}(n), y_{2}(n), \ldots\right\}$.

(2) $\Rightarrow$ (1). В силу теоремы 1 , нам достаточно доказать, что счетная система $L=\left\{x e_{i}=a_{i} e_{i}\right\}_{i=1}^{\infty}$ конечно разрешима. Это равносильно тому, что конечная система $L_{n}=\left\{x e_{i}=a_{i} e_{i}\right\}_{i=1}^{n}$ разрешима для любого $n$. Так как все идемпотенты $e_{i}$ ортогональны, то $\sum_{i=0}^{n} a_{i} e_{i}$ - частное решение системы $L_{n}$.

\section{Список литературы}

1. Каш Ф., Модули и кольц̧а. Мир, Москва, 1981.

2. Фейс К., Алгебра: кольияа, модули и категории, 2. Мир, Москва, 1979.

3. Goodearl K. R., Von Neumann regular rings. Pitman, London, 1979.

4. Osofsky B. L., Rings all of whose finitely generated modules are injective. Pacific J. Math. (1964) 14, 645-650.

Статья поступила 16.06.2009. 